We appreciate the response to this publication feature and welcome all contributions. Contributions may be sent to José A. Mascorro, our Technical Editor, at his e-mail address: jmascor@tulane.edu. José may also be reached at the Department of Structural and Cellular Biology,

Tulane University Health Sciences Center, 1430 Tulane Ave., New Orieans, LA 70112 and Ph: (504) 584-2747 Fax:

\section{Support Films with Uniform Hole Size}

Kenneth $\mathrm{H}$. Downing

Lawrence Berkeley National Laboratory kdowning@lbl.gov

Holey carbon films mounted on standard EM grids are routinely used as specimen supports in electron cryo-microscopy. Adroplet of the sample containing, for example, a suspension of virus particles or protein molecules, is applied to the grid, blotted to a thin layer and rapidly frozen. The particles are then imaged where the liquid spans the holes, without interference from an underlying carbon support film. Many recipes for holey films have been presented in the literature, generally employing some method of forming bubbles in a layer of solvent in which a material is dissolved that then forms a discontinuous layer as the solvent evaporates. The methods in use generally produce a wide range of hole sizes and suffer from a large degree of variability. The hole size and distribution often depends on environmental conditions such as humidity. With practice and luck, one can usually adjust the conditions to obtain the desired results. However, a method that would always produce the

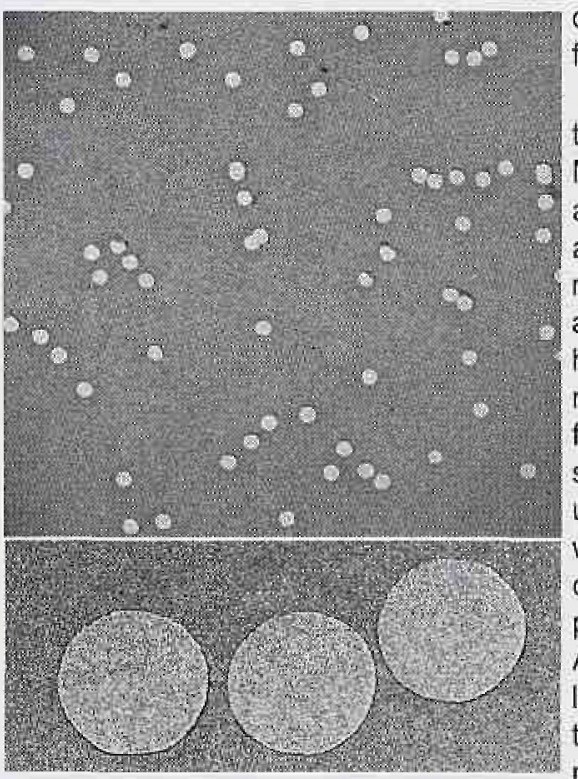
desired holes would be a frequent advantage.

Here is a method that derives from Martin Muller and Theo Koller at the ETH in Zurich and produces a very nice distribution of holes all of the same size. The holey films are made as replicas of Nucleopore $B$ filters, which have a very smooth surface with uniform, round holes with a diameter that can be chosen for the particular application. A water-soluble parting layer is evaporated onto the filter before evaporating the carbon film,

which is then floated off and mounted onto the grids in the normal way. We use Whatman Nucleopore $\circledast$ Track-Etch Membrane filters. We find that with a nominal cutoff of $2.0 \mu \mathrm{m}$ (cat. No. 110611 for 25 $\mathrm{mm}$ diameter filters) the hole size is about $1.5 \mu \mathrm{m}$. The filters are taped (using a water-resistant tape) onto a glass microscope slide. The parting layer is sodium metaphosphate, which is available as Victawet from EM suppliers. A bit of the material about the size of a grain of rice is placed in a tungsten basket in an evaporator and heated slowly to evaporate. The Victawet tends to bubble and outgas as it warms, so slow heating is necessary to make sure that it doesn't jump out of the basket. Once the material is all evaporated, carbon is evaporated to the desired thickness. It is a good idea to have both the Victawet and carbon sources placed as close together as possible directly above the filter, in order to avoid forming a film along the inside edge of the holes in the plastic film.
The film should then float off onto a water surface as the slide is slowly submerged.

Holey films made in this way may not have as many holes as those made by other methods, but all the holes are the right size. They are free from the plastic residue that may be left from other methods, and the holes have little or no collar around the edge that can adversely affect the ice thickness. These grids are a very effective intermediate between holey films made with the various solvent techniques, which produce random hole sizes, and commercial Quantifoil@ grids, which have uniform holes on a regular lattice. ㅁ.

\section{Cleaning a Cold Cathode Gauge Tube} Owen P. Mills

Michigan Technological University opmills@mtu.edu

This topic appeared on the MSA list server in December 2002 and January 2003 . While cleaning my gauge tube recently, I documented the steps. In this article I describe cleaning a Varian 524-2 gauge tube. There are a number of other cold cathode and penning style gauge tubes on the market, Edwards CP-25 (old penning), CVC GPH-001A (old cold cathode), Pfeiffer IRK 250 (new cold cathode) to name a few. The cleaning steps are very similar. Consult the instructions that came with the gauge tube or visit the vendor's websites.

First, when to clean: I pull the tube to check for cleanliness when the vacuum readings begin to wander erratically up and down scale. When I decided to pull the gauge tube on my evaporator, the readings ranged between $1 \times 10^{-5}$ and $9 \times 10^{-5}$. The meter would not remain steady.

Routine use eventually will result in the need to clean a gauge tube. In evaporators, pumping wet, volatile samples and substances can adversely influence cleanliness. Older systems required that users turn the high vacuum controller on and off. Leaving the controller on when the vacuum is below the recommended level $\left(1 \times 10^{-2}\right.$ for the Varian 524) for the cold cathode gauge quickly leads to contamination. You can minimize the frequency of required cleaning by having your gauge electronics shut off when the vacuum drops below $10^{-4}$. I added a vacuum switch to my old Denton evaporator for that purpose.

Removing the gauge tube: Removing the gauge depends on the type of system it is connected to. Ensure that you have properly vented the vacuum system before attempting to remove the gauge tube. CAUTION: the cold cathode gauge is operated by applying a high voltage, up to 4000 VDC, between the anode and cathode (Bigelow). Be sure you have disconnected the control unit before removing the cables to the gauge.

Cleaning: If you utilize a Varian gauge tube, the company makes a convenient kit that includes a few spare parts for disassembling the gauge (Fig. 1). The disassembled gauge is shown in

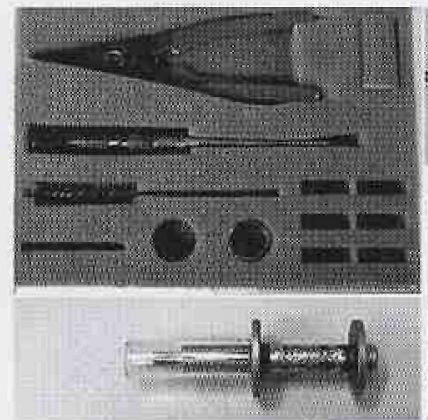

Fig. 3, Dirty anode

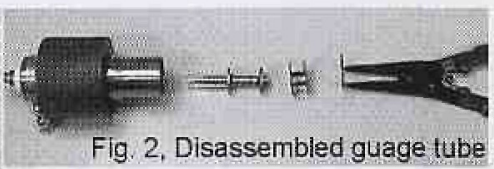

Fig. 1, Varian tool kit

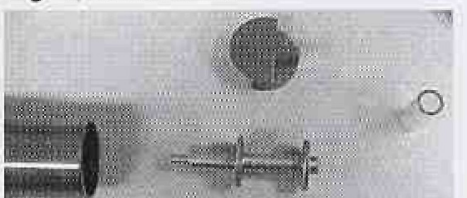

Fig. 4, Bead blasted clean anode 